\title{
Transient Vision Loss - A Rare Oxaliplatin-Induced Ophthalmologic Side Effect: A Report of Two Cases
}

\author{
Loic Ah-Thiane ${ }^{a}$ Jean-Luc Raoul ${ }^{a}$ Sandrine Hiret ${ }^{a}$ Hélène Senellart ${ }^{a}$ \\ Frédéric Dumont $^{\mathrm{b}}$ Judith Raimbourg ${ }^{\mathrm{a}}$ \\ aDepartment of Medical Oncology, Institut de Cancérologie de I'Ouest, Saint-Herblain, \\ France; 'bepartment of Surgical Oncology, Institut de Cancérologie de l'Ouest, Saint- \\ Herblain, France
}

\section{Keywords}

Oxaliplatin · Sight loss · Acute ocular toxicity · Optic neuritis

\begin{abstract}
Oxaliplatin, a platinum-based chemotherapeutic agent, is responsible for induced peripheral sensory neuropathy. Only a few cases of ophthalmologic toxicity have been reported. We report here two cases of sudden transient vision loss after oxaliplatin administration, in one case intraperitoneally. These symptoms likely reflect optic neuritis that could be included in the spectrum of oxaliplatin-induced neuropathy.

\section{Introduction}

Oxaliplatin is a platinum-based chemotherapy often used in digestive cancers, and particularly in the adjuvant setting of colorectal cancers. Oxaliplatin has been associated with different side effects: hematological, gastrointestinal or hypersensitivity effects (including severe anaphylaxis), and notably peripheral neuropathy [1]. Such peripheral neuropathy is very frequent and represented by two distinct forms: an acute, cold-induced form with paresthesia and muscle spasms affecting at least $80 \%$ of oxaliplatin-treated patients, and a chronic, cumulative, dose-related form [2] corresponding to chronic, pure sensory axonal neuropathy [3]. In addition to this frequently described toxicity, a less common side effect is ophthalmologic toxicity. We report here two cases of ophthalmologic toxicities observed in our institution during oxaliplatin treatment for colorectal cancer.

\section{Karger $\stackrel{\text { ! }}{=}$}




\section{Case Reports in Oncology}

\begin{tabular}{l|l}
\hline Case Rep Oncol 2021;14:483-486 \\
\hline DOI: 10.1159/000514656 & $\begin{array}{l}\text { @ 2 2021 The Author(s). Published by S. Karger AG, Basel } \\
\text { www.karger.com/cro }\end{array}$ \\
\hline
\end{tabular}

Ah-Thiane et al.: Transient Vision Loss - A Rare Oxaliplatin-Induced Ophthalmologic Side Effect: A Report of Two Cases

\section{Case Reports}

Case 1

In February 2013, a 50-year-old woman was hospitalized for headache and nausea revealing deep cerebral vein thrombosis (CT scan, MRI). Anticoagulants were initiated and all signs disappeared within a few days. In April, while the patient was still under low-molecularweight heparin, anemia led to the diagnosis of right colon cancer with peritoneal carcinosis. After a multidisciplinary team meeting, it was decided to propose three cycles of chemotherapy, and then to include her in a trial comparing complete surgery (colic and peritoneal) alone or associated with hyperthermic intraperitoneal chemotherapy (PRODIGE 7 trial EudraCT number: 2006-006175-20). Two days after the first cycle of FOLFOX (April 2013), she experienced acute bilateral sight loss episodes (6-10 episodes per day, lasting some minutes), spontaneously resolving within a week and not associated with other severe peripheral neuropathy, particularly with only minor paresthesia. Cerebral imaging (improvement of the cerebral vein thrombosis) and ophthalmologic examination were normal. Oxaliplatin was then discontinued, and the second and third cycles (5-FU alone) were well tolerated with no visual side effect. In June 2013, she underwent R0 surgery (right colectomy, complete surgical resection of the peritoneal carcinomatosis) and HIPEC with oxaliplatin $\left(360 \mathrm{mg} / \mathrm{m}^{2}\right)$. Two days after surgery, while the postoperative period was uneventful, she had novel episodes of transient vision loss disappearing 2 days later. On pathological examination, the tumor was classified as T4bN0 ( $0 / 16$ nodes harvested) with peritoneal carcinomatosis (M1); the tumor was MSI. No medical treatment was proposed and the follow-up is ongoing, with no tumor recurrence or new ophthalmologic sign in November 2020.

Case 2

In 2019, a 57-year-old nurse had abdominal pain and discomfort. Her past history was marked by ophthalmologic problems with congenital cataract and retinal detachment, both requiring many eye surgeries. Colonoscopy revealed a sigmoid mass and she underwent a left colectomy in April 2019. Pathological examination concluded in adenocarcinoma classified as pT4aN2b (7 positive nodes among 17 examined) M0, resection R0. She was included in an adjuvant chemotherapy trial comparing FOLFOX to FOLFIRINOX (IROCAS trial, EUDRACT number: 2016-001491-29). After the first cycle of FOLFIRINOX, she experienced severe diarrhea leading to a decrease in the irinotecan dose. Despite this decrease, she had another episode of severe diarrhea following the second cycle and irinotecan was stopped. Therefore, the third cycle was limited to FOLFOX. From the second day after this third cycle, she started to experience sight loss limited to the right eye, lasting some seconds but occurring repeatedly until the fourth day. Of note, she did not experience dysesthesia or muscle cramps. A complete ophthalmologic workup was done by her usual ophthalmologist and everything was normal. Oxaliplatin was then definitively stopped and she continued a 5FU regimen until the twelfth cycle with no recurrence of symptoms. In November 2020, she had no sign of tumor recurrence.

\section{Discussion}

These two cases describe acute, transient, and reversible ophthalmologic toxicity induced by oxaliplatin as reversible acute sight loss, including one case following intraperitoneal use.

Acute oxaliplatin-induced peripheral neuropathy is observed in most patients receiving oxaliplatin, with cold-triggered distal paresthesia and pharyngo-laryngeal dysesthesia

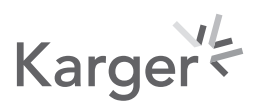


causing a feeling of difficulty breathing; these sensory symptoms are sometimes paralleled by motor symptoms including muscle fasciculation and cramps. The symptoms, occurring during or shortly after oxaliplatin infusion, are usually transient and mild [4]. It has been reported that patients who had severe acute toxicities will develop more severe chronic neurotoxicity [2]. Chronic neurotoxicity is frequent and may markedly impair quality of life. This cumulative neurotoxicity has led researchers to conduct a large-scale randomized trial comparing 3 to 6 months of adjuvant therapy to oxaliplatin-containing chemotherapy [5]. If chronic neurotoxicity seems related to damage to dorsal root ganglia neurons, the acute symptoms are due to oxaliplatin's effects on voltage-gated sodium channel kinetics [6].

Oxaliplatin-related ocular toxicity seems very rare with only a few cases reported.

A 52-year-old woman had, after three cycles of $5 \mathrm{FU}$ plus oxaliplatin chemotherapy, visual changes with blurred and altered color vision associated concomitantly with usual paresthesia and dysesthesia. On ophthalmologic examination, she had some abnormalities including severe bilateral concentric scotoma with mild central defect, and a diminished response on electrooculogram. Oxaliplatin was stopped, and 3 weeks later the visual disturbances improved until complete clinical resolution, but electrooculogram remained abnormal [7]. A second case was a 71-year-old man treated with the FOLFIRINOX regimen for pancreatic adenocarcinoma. Immediately after oxaliplatin injections for the first and second cycles, the patient presented with visual changes: complete loss of vision from one eye followed by tunnel vision that fully resolved within 2 days after the first cycle and 5 days after the third. A complete ophthalmologic examination was negative. The FOLFIRINOX regimen was then stopped and no further abnormalities were reported [8]. In another case, the troubles began with peripheral vision loss progressing to the central vision with bilateral papilledema on fundoscopy. The same authors also report two other cases of tunnel vision and one of complete sight loss [9].

The ophthalmologic disorders presented in our clinical cases and in the literature show similarities with acute peripheral neuropathy: quick onset, transient toxicity, increased severity with treatment continuation, and disappearance after treatment discontinuation. The onset of toxicity after intraperitoneal injection is explained by a similar blood concentration of oxaliplatin as following intravenous injection [10].

All these cases reported similar symptoms (sight loss, tunnel vision, or color changes) seeming to correspond to optic neuritis; normal funduscopic examination or signs of papilledema in more marked cases [9] are consistent with optic neuritis.

\section{Conclusion}

In conclusion, our two clinical cases describe a rare ophthalmic manifestation of oxaliplatin-induced acute peripheral neuropathy linked to possible minor optic neuritis; these symptoms were transient and reversible in our cases, as well as in those from the literature, after definitively stopping oxaliplatin. Oxaliplatin-related sight disorders should be recognized promptly by health professionals because, even if the signs are mostly transient, delayed diagnosis and treatment continuation could lead to irreversible sequela to the optic nerve [11].

\section{Karger'k}




\section{Case Reports in Oncology}

\begin{tabular}{l|l}
\hline Case Rep Oncol 2021;14:483-486 \\
\hline DOI: 10.1159/000514656 & $\begin{array}{l}\text { @ 2021 The Author(s). Published by S. Karger AG, Basel } \\
\text { www.karger.com/cro }\end{array}$ \\
\hline
\end{tabular}

Ah-Thiane et al.: Transient Vision Loss - A Rare Oxaliplatin-Induced Ophthalmologic Side Effect: A Report of Two Cases

\section{Statement of Ethics}

The authors state that the subjects gave their written informed consent to their case being published and that the research was conducted ethically in accordance with the World Medical Association Declaration of Helsinki.

\section{Conflict of Interest Statement}

The authors have no conflicts of interest to declare.

\section{Funding Sources}

None.

\section{Author Contributions}

L.A.-H. and J.-L.R.: manuscript writing; L.A.-H., J.-L.R., J.R.: data analysis and interpretation; S.H., H.S., F.D., and J.R.: data curation. Review, final approval, and editing: all authors.

\section{References}

1 Becouarn Y, Ychou M, Ducreux M, et al. Phase II trial of oxaliplatin as first-line chemotherapy in metastatic colorectal cancer patients. Digestive Group of French Federation of Cancer Centers. J Clin Oncol. 1998;16: 2739-44.

2 Pachman DR, Qin R, Seisler DK, Smith EM, Beutler AS, Ta LE, et al. Clinical Course of Oxaliplatin-Induced Neuropathy: Results From the Randomized Phase III Trial N08CB (Alliance). J Clin Oncol. 2015;33(30):341622.

3 Park SB, Lin CS, Krishnan AV, Goldstein D, Friedlander ML, Kiernan MC. Oxaliplatin-induced neurotoxicity: changes in axonal excitability precede development of neuropathy. Brain. 2009;132(Pt 10):2712-23.

4 Saif MW, Reardon J. Management of oxaliplatin-induced peripheral neuropathy. Ther Clin Risk Manag. 2005; 1(4):249-58.

5 Grothey A, Sobrero AF, Shields AF, Yoshino T, Paul J, Taieb J, et al. Duration of Adjuvant Chemotherapy for Stage III Colon Cancer. N Engl J Med. 2018;378(13):1177-88.

6 Sittl R, Lampert A, Huth T, Schuy ET, Link AS, Fleckenstein J, et al. Anticancer drug oxaliplatin induces acute cooling-aggravated neuropathy via sodium channel subtype $\mathrm{Na}(\mathrm{V}) 1.6$-resurgent and persistent current. Proc Natl Acad Sci USA. 2012;109(17):6704-9.

7 Mesquida M, Sanchez-Dalmau B, Ortiz-Perez S, Pelegrín L, Molina-Fernandez JJ, Figueras-Roca M, et al. Oxaliplatin-Related Ocular Toxicity. Case Rep Oncol. 2010;3(3):423-7.

8 Noor A, Desai A, Singh M. Reversible Ocular Toxicity of Oxaliplatin: A Case Report. Cureus. 2019;11(5):e4582.

9 O’Dea D, Handy CM, Wexler A. Ocular changes with oxaliplatin. Clin J Oncol Nurs. 2006;10(2):227-9.

10 Dumont F, Passot C, Raoul JL, Kepenekian V, Lelièvre B, Boisdron-Celle M, et al. A phase I dose-escalation study of oxaliplatin delivered via a laparoscopic approach using pressurised intraperitoneal aerosol chemotherapy for advanced peritoneal metastases of gastrointestinal tract cancers. Eur J Cancer. 2020;140:37-44.

11 Cavaletti G, Marmiroli P. Management of Oxaliplatin-Induced Peripheral Sensory Neuropathy. Cancers (Basel). $2020 ; 12(6)$. 